TRANSACTIONS OF THE

AMERICAN MATHEMATICAL SOCIETY

Volume 360, Number 5, May 2008, Pages 2265-2284

S 0002-9947(07)04425-X

Article electronically published on December 20, 2007

\title{
THE BROWN-PETERSON COHOMOLOGY OF THE CLASSIFYING SPACES OF THE PROJECTIVE UNITARY GROUPS $P U(p)$ AND EXCEPTIONAL LIE GROUPS
}

\author{
MASAKI KAMEKO AND NOBUAKI YAGITA
}

\begin{abstract}
For a fixed prime $p$, we compute the Brown-Peterson cohomologies of classifying spaces of $P U(p)$ and exceptional Lie groups by using the Adams spectral sequence. In particular, we see that $B P^{*}(B P U(p))$ and $K(n)^{*}(B P U(p))$ are even dimensionally generated.
\end{abstract}

\section{INTRODUCTION}

Let $p$ be a fixed odd prime and denote by $B P^{*}(X)$ ( resp. $\left.P(m)^{*}(X)\right)$ the BrownPeterson cohomology of a space $X$ with the coefficient ring $B P^{*}=\mathbb{Z}_{(p)}\left[v_{1}, v_{2}, \cdots\right]$ (resp. $P(m)^{*}=\mathbb{Z} / p\left[v_{m}, v_{m+1}, \cdots\right]$ ) where $\operatorname{deg} v_{k}=-2 p^{k}+2$. We denote by $P U(n)$ the projective unitary group which is the quotient of the unitary group $U(n)$ by its center $S^{1}$. Recall that the cohomologies of $P U(p)$ and the exceptional Lie groups $F_{4}, E_{6}, E_{7}, E_{8}$ have odd torsion elements. In this paper, we compute the Brown-Peterson cohomologies of classifying spaces $B G$ of these Lie groups $G$ as $B P^{*}$-modules using the Adams spectral sequence. Let us write $H^{*}(X ; \mathbb{Z} / p)$ by simply $H^{*}(X)$ and let $\mathcal{A}$ be the $\bmod p$ Steenrod algebra.

Our main result is as follows:

Theorem 1.1. Let $(G, p)$ be one of cases $(G=P U(p), p)$ for an arbitrary odd prime $p$ and $G=F_{4}, E_{7}$ for $p=3$, and $G=E_{8}$ for $p=5$. Then the $E_{2}$-terms of the Adams spectral sequences abutting to $B P^{*}(B G)$ and $P(m)^{*}(B G)$ for $m \geq 1$,

$$
\operatorname{Ext}_{\mathcal{A}}^{s, t}\left(H^{*}(B P), H^{*}(B G)\right), \quad \operatorname{Ext}_{\mathcal{A}}^{s, t}\left(H^{*}(P(m)), H^{*}(B G)\right),
$$

have no odd degree elements.

An immediate consequence is as follows:

Corollary 1.2. For $(G, p)$ in Theorem 1.1, the Adams spectral sequences abutting to $B P^{*}(B G)$ and $P(m)^{*}(B G)$ in the previous theorem collapse at the $E_{2}$-level. In particular $B P^{\text {odd }}(B G)=P(m)^{\text {odd }}(B G)=0$.

Recall $K(m)^{*}(X) \cong K(m)^{*} \otimes_{P(m)^{*}} P(m)^{*}(X)$ is the Morava $K$-theory. From the above theorem and corollary, we see $K(m)^{\text {odd }}(B P U(p))=0$. Then we have the following corollary ([Ko-Ya], [Ra-Wi-Ya]).

Received by the editors November 2, 2005.

2000 Mathematics Subject Classification. Primary 55P35, 57T25; Secondary 55R35, 57T05.

Key words and phrases. BP-theory, $P U(p)$, exceptional Lie groups. 
Corollary 1.3. For $(G, p)$ in Theorem 1.1, the following holds:

(1) $B P^{*}(B G)$ is $B P^{*}$-flat for $B P^{*}(B P)$-modules, i.e., $B P^{*}(B G \times X) \cong$ $B P^{*}(B G) \otimes_{B P^{*}} B P^{*}(X)$ for all finite complexes $X$.

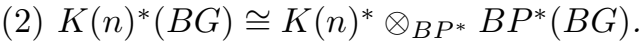

(3) $P(n)^{*}(B G) \cong P(n)^{*} \otimes_{B P^{*}} B P^{*}(B G)$.

We give the $B P^{*}$-module structure of $B P^{*}(B P U(p))$ more explicitly in this introduction. For the exceptional Lie group cases, the explicit formulas are given in $\S 5$.

Theorem 1.4. There exists a $B P^{*}$-algebra exact sequence

$$
0 \rightarrow B P^{*} \widehat{\otimes} M \rightarrow g r B P^{*}(B P U(p)) \rightarrow B P^{*} \widehat{\otimes} I N /\left(f_{0}, f_{1}\right) \rightarrow 0
$$

where

(1) $M \cong \mathbb{Z}_{(p)}\left[x_{4}, x_{6}, \ldots, x_{2 p}\right]$ as $\mathbb{Z}_{(p)}$-modules (but not $\mathbb{Z}_{(p)}$-algebras).

(2) $I N \cong \mathbb{Z}_{(p)}\left[x_{2 p+2}, x_{2 p(p-1)}\right]\left\{x_{2 p+2}\right\}$, the principal ideal of $\mathbb{Z}_{(p)}\left[x_{2 p+2}, x_{2 p(p-1)}\right]$ generated by $x_{2 p+2}$.

(3) Relations $f_{0}, f_{1}$ are given with modulo $\left(p, v_{1}, v_{2}, \cdots\right)^{2}$ :

$$
f_{0} \equiv v_{0}-v_{2} x_{2 p+2}^{p-1}+\cdots, \quad f_{1} \equiv v_{1}-v_{2} x_{2 p(p-1)}+\cdots .
$$

Remark. In the above theorem, subscript $i$ of $x_{i}$ means its degree. The algebra $B P^{*}(B P U(p))$ does not contain the subalgebra $B P^{*} \widehat{\otimes} \mathbb{Z}_{(p)}\left[x_{4}, \ldots, x_{2 p}\right]$, but contains a subalgebra which is isomorphic as $B P^{*}$-modules to the above $B P^{*}$ subalgebra.

For an algebraic group $G$ over $\mathbb{C}$, Totaro [To] defines its Chow ring and conjectures that $B P^{*}(B G) \otimes_{B P^{*}} \mathbb{Z}_{(p)} \cong C H^{*}(B G)_{(p)}$. Recall that $P G L(p, \mathbb{C})$ is the algebraic group over $\mathbb{C}$ corresponding to the Lie group $P U(p)$.

Theorem 1.5. There exists an isomorphism

$$
B P^{*}(B P U(p)) \otimes_{B P^{*}} \mathbb{Z}_{(p)} \cong C H^{*}(B G L(p, \mathbb{C}))_{(p)} .
$$

Hence there exists an additive isomorphism

$$
C H^{*}(B G L(p, \mathbb{C}))_{(p)} \cong \mathbb{Z}_{(p)}\left[x_{4}, x_{6}, \cdots, x_{2 p}\right] \oplus \mathbb{F}_{p}\left[x_{2 p+2}, x_{2 p(p-1)}\right]\left\{x_{2 p+2}\right\} .
$$

Remark. Vistoli [Vi] also determined the additive structure of the Chow ring and integral cohomology of $B P G L(p, \mathbb{C})$ by using stratified methods of Vezzosi [Ve] (see also $[\mathrm{Mo}-\mathrm{Vi}])$. Moreover he shows that for $G=P G L(p, \mathbb{C})$,

$$
H^{*}(G ; \mathbb{Z}) \rightarrow H^{*}(B T ; \mathbb{Z})^{W_{G}(T)}
$$

is epic.

Let $M G L^{*, *}(X)$ be the motivic cobordism ring defined by V. Voevodsky [Vo1] and $M G L^{2 *, *}(X)=\bigoplus_{i} M G L^{2 i, i}(X)$.

Corollary 1.6. $M G L^{2 * * *}(B P G L(p, \mathbb{C}))_{(p)} \cong M U^{*}(B P U(p))_{(p)}$.

We prove Theorem 1.1 by using the Adams spectral sequence converging to the Brown-Peterson cohomology. The $E_{1}$-term of the spectral sequence could be given by

$$
\mathbb{F}_{p}\left[v_{0}, v_{1}, \cdots\right] \widehat{\otimes} H^{*}(X) \quad \text { with } \quad d_{1} x=\sum_{k=0}^{\infty} v_{k} Q_{k} x
$$


where the $Q_{k}$ 's are Milnor's operations. (Here we identify $\mathbb{F}_{p}\left[v_{0}=p, v_{1}, \cdots\right]=$ $P(0)^{*}$.)

By the change-of-rings isomorphism, the $E_{2}$-term is

$$
\operatorname{Ext}_{\mathcal{A}}\left(H^{*}(B P), H^{*}(X)\right) \cong \operatorname{Ext}_{\mathcal{E}}\left(\mathbb{F}_{p}, H^{*}(X)\right)
$$

where $\mathcal{E}=\Lambda\left(Q_{0}, Q_{1}, \cdots\right)$. The $E_{\infty}$-term is given by $\operatorname{gr} B P^{*}(X)$.

To state the cohomology $H^{*}(B P U(p))$, we recall the Dickson algebra. Let $A_{n}$ be an elementary abelian $p$-group of rank $n$, and

$$
H^{*}\left(B A_{n}\right) \cong \mathbb{F}_{p}\left[t_{1}, \ldots, t_{n}\right] \otimes \Lambda\left(d t_{1}, \ldots, d t_{n}\right) \quad \text { with } \beta\left(d t_{i}\right)=t_{i} .
$$

The Dickson algebra is

$$
D_{n}=\mathbb{F}_{p}\left[t_{1}, \ldots, t_{n}\right]^{G L\left(n, \mathbb{F}_{p}\right)} \cong \mathbb{F}_{p}\left[c_{n, 0}, \ldots, c_{n, n-1}\right]
$$

with $\left|c_{n, i}\right|=2\left(p^{n}-p^{i}\right)$. The invariant ring under $S L\left(n, \mathbb{F}_{p}\right)$ is also given:

$$
S D_{n}=\mathbb{F}_{p}\left[t_{1}, \ldots, t_{n}\right]^{S L\left(n, \mathbb{F}_{p}\right)} \cong D_{n}\left\{1, e_{n}, \ldots, e_{n}^{p-2}\right\} \quad \text { with } e_{n}^{p-1}=c_{n, 0} .
$$

We also recall Mui's ([Mu]) result by using $Q_{i}$ according to Kameko and Mimura $[\mathrm{Ka}-\mathrm{Mi}]$

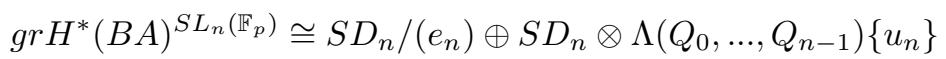

where $u_{n}=d t_{1} \ldots d t_{n}$ and $e_{n}=Q_{0} \ldots Q_{n-1} u_{n}$.

Theorem 1.7. There exists a short exact sequence

$$
0 \rightarrow M / p \rightarrow H^{*}(B P U(p)) \rightarrow N \rightarrow 0
$$

where $M / p$ is the trivial $\mathcal{E}$-module given in Theorem 1.4 and

$$
N=S D_{2} \otimes \Lambda\left(Q_{0}, Q_{1}\right)\left\{u_{2}\right\} \cong \mathbb{F}_{p}\left[x_{2 p+2}, x_{2\left(p^{2}-p\right)}\right] \otimes \Lambda\left(Q_{0}, Q_{1}\right)\left\{u_{2}\right\}
$$

identifying $x_{2 p+2}=e_{2}$ and $x_{2\left(p^{2}-p\right)}=c_{2,1}$.

This theorem is proved by using the following facts. The group $G=P U(p)$ has just two conjugacy classes of maximal elementary abelian $p$-subgroups, one of which is toral and the other is nontoral $A$ of $\operatorname{rank}_{p}=2$. The cohomology $H^{*}(B G)$ is detected by these two subgroups. The restriction image to the nontoral subgroup is $i_{A}^{*}\left(H^{*} P U(p)\right) \cong H^{*}(B A)^{S L\left(2, \mathbb{F}_{p}\right)}$. Similar (but not the same) facts also hold for the exceptional Lie groups given in Theorem 1.1.

The algebraic main result in this paper is as follows:

Theorem 1.8. For $m \geq 0$, define $f_{0}, \ldots, f_{n-1}$ in $P(m)^{*} \widehat{\otimes} S D_{n}$ by

$$
d_{1} u_{n}=\sum_{k \geq m} v_{k} Q_{k}\left(u_{n}\right)=f_{0} Q_{0} u_{n}+\cdots+f_{n-1} Q_{n-1} u_{n} .
$$

Then the sequence $f_{0}, \ldots, f_{n-1}$ is a regular sequence in $P(m)^{*} \widehat{\otimes} S D_{n}$.

With the notation in this theorem, we prove that the complex

$$
C=\left(P(m)^{*} \widehat{\otimes} S D_{n} \otimes \Lambda\left(Q_{0}, Q_{1}, \ldots, Q_{n-1}\right)\left\{u_{n}\right\}, d_{1}\right)
$$

with the differential $d_{1} u_{n}=\sum_{i=0}^{n-1} f_{i} Q_{i} u_{n}$ is a Koszul complex. This means that

$$
H_{i}\left(C, d_{1}\right)=\left\{\begin{array}{l}
P(m)^{*} \widehat{\otimes} S D_{n}\left\{e_{n}\right\} /\left(f_{0}, \ldots, f_{n-1}\right) \quad \text { for } i=0 \\
0 \quad \text { for } i \geq 1 .
\end{array}\right.
$$

Thus Theorem 1.1 follows from the above theorem. 
Remark about the convergence of the Adams spectral sequence. By Theorem 15.6 in Boardman's paper [Bo2], since $H^{*}(B P)$ is of finite type, the above Adams spectral sequence is conditionally convergent. Moreover, since we prove the above Adams spectral sequence collapses at the $E_{2}$-level, by the remark after Theorem 7.1 in [Bo1], the above Adams spectral sequence is strongly convergent, so that we know the Brown-Peterson cohomology up to group extension.

We now give an outline of the paper. In $\S 2$, we note the relation of the Koszul complex and the $E_{1}$-term of the Adams spectral sequence for $\Lambda\left(Q_{0}, \ldots, Q_{n-1}\right)$-free $H^{*}(X)$. Section 3 is stated about the Dickson invariant rings and the Milnor $Q_{i^{-}}$ operation on the cohomology of classifying spaces of elementary abelian $p$-groups. In $\S 4$, we compute $H^{*}(B G), M U^{*}(B G), C H^{*}(B G)$ for $G=P U(p)$. The similar computations are done in $\S 5$ for exceptional groups. The last section is devoted to the proof of Theorem 1.8.

\section{The Koszul complex and the Adams spectral Sequence}

For spaces $X, Y$, the Adams spectral sequence converging to the stable homotopy $\{X, Y\}$ has the $E_{2}$-term

$$
E_{2}^{*, *} \cong \operatorname{Ext}_{\mathcal{A}}\left(H^{*}(Y), H^{*}(X)\right) \Longrightarrow\{X, Y\} .
$$

We want to consider the case $Y=P(m)$.

Recall that $\mathcal{A} \cong H^{*}(B P) \otimes \mathcal{E}$ with $\mathcal{E}=\Lambda\left(Q_{0}, Q_{1}, \ldots\right)$. Similarly $H^{*}(P(m)) \cong$ $H^{*}(B P) \otimes \Lambda\left(Q_{0}, \ldots, Q_{m-1}\right)$. Hence the $E_{2}$-term of the Adams spectral sequence abutting to $P(m)^{*}(X)$ is given by

$$
\begin{gathered}
\operatorname{Ext}_{\mathcal{A}}\left(H^{*}(P(m)), H^{*}(X)\right) \cong \operatorname{Ext}_{H^{*}(B P) \otimes \mathcal{E}}\left(H^{*}(B P) \otimes \Lambda\left(Q_{0}, \ldots, Q_{m-1}\right), H^{*}(X)\right) \\
\cong \operatorname{Ext}_{\mathcal{E}_{m}}\left(\mathbb{F}_{p}, H^{*}(X)\right) \quad \text { where } \mathcal{E}_{m}=\Lambda\left(Q_{m}, Q_{m+1}, \cdots\right) .
\end{gathered}
$$

We will study the case

$$
0 \rightarrow M \rightarrow H^{*}(X) \rightarrow N \rightarrow 0
$$

where $M$ is $\mathcal{E}$-trivial and $N$ is a free $\Lambda\left(Q_{0}, \ldots, Q_{n-1}\right)$-module having the following properties. (For example, $X=B P U(p)$; recall Theorem 1.7.)

For a $\mathbb{Z} / p$-algebra $S$, suppose that $\mathcal{E}$ acts on

$$
N=S \otimes \Lambda\left(Q_{0}, \ldots, Q_{n-1}\right)
$$

such that $Q_{i}$ acts as a derivation and $Q_{n}(s \otimes t)=s \otimes Q_{i}(t)$ for $s \in S, t \in N$. Then for each $k \geq 0$, there exist $\alpha_{0, k}, \ldots, \alpha_{n-1, k} \in S$ such that

$$
Q_{k} x=\alpha_{0, k} Q_{0} x+\cdots+\alpha_{n-1, k} Q_{n-1} x
$$

for all $x \in N$ (compare the proof of Proposition 3.3 below).

Let $R=P(m)^{*} \widehat{\otimes} S$. For $i=0, \ldots, n-1$, let

$$
f_{i}=v_{m} \alpha_{i, m}+v_{m+1} \alpha_{i, m+1}+\cdots \in R .
$$

The derived functor $\operatorname{Ext}_{\mathcal{E}_{m}}\left(\mathbb{F}_{p}, N\right)$ is the cohomology of a cochain complex

$$
E_{1}=P(m)^{*} \widehat{\otimes} N=R \otimes \Lambda\left(Q_{0}, \ldots, Q_{n-1}\right)
$$

with a differential

$$
d_{1}(x)=\sum_{k \geq m} v_{k} Q_{k}(x)=f_{0} Q_{0}(x)+\cdots+f_{n-1} Q_{n-1}(x) .
$$


Proposition 2.1. With the notation above, if $f_{0}, \ldots, f_{n-1}$ is a regular sequence in $R=P(m)^{*} \widehat{\otimes} S$, then

$$
\operatorname{Ext}_{\mathcal{A}}\left(H^{*}(P(m), N)=\operatorname{Ext}_{\mathcal{E}_{m}}\left(\mathbb{F}_{p}, N\right) \cong R /\left(f_{0}, \ldots, f_{n-1}\right) .\right.
$$

Hence Theorem 1.1 (for $G=P U(p)$ ) follows from Theorem 1.7, 1.8 and the above proposition. (We will show Theorem 1.7 in $\S 4$ and Theorem 1.8 in $\S 3,6$.)

To see the above proposition, we recall the Koszul complex. Let $R$ be a commutative algebra and let $g_{0}, \ldots, g_{n-1}$ be a sequence of $R$. Let $J$ be the ideal of $R$ generated by $g_{0}, \ldots, g_{n-1}$. Let $\Lambda^{r}=\Lambda^{r}\left(x_{0}, \ldots, x_{n-1}\right)$ be $r$-homogeneous parts with $\operatorname{deg}\left(x_{i}\right)=1$. The Koszul complex is a chain complex

$$
0 \rightarrow R \otimes \Lambda^{n} \stackrel{d}{\longrightarrow} \cdots \rightarrow R \otimes \Lambda^{1} \stackrel{d}{\longrightarrow} R \otimes \Lambda^{0} \rightarrow 0
$$

with the differential

$$
d\left(x_{i_{1}} \cdots x_{i_{r}}\right)=\sum_{j=1}^{r}(-1)^{j+1} g_{i_{j}} x_{i_{1}} \cdots \widehat{x}_{i_{j}} \cdots x_{i_{r}} .
$$

Let us denote by $H_{k}\left(R \otimes \Lambda^{*}, d\right)$ the induced homology. The following proposition is well known.

Proposition 2.2. If $g_{0}, \ldots, g_{n-1}$ is a regular sequence in $R$, then

$$
H_{k}\left(R \otimes \Lambda^{*}, d\right) \cong\left\{\begin{array}{l}
R / J \quad \text { for } k=0, \\
0 \quad \text { for } k \geq 1 .
\end{array}\right.
$$

Proof of Proposition 2.1. Let $g_{i}=(-1)^{i} f_{i}$. It is clear that $g_{0}, \ldots, g_{n-1}$ is a regular sequence if and only if $f_{0}, \ldots, f_{n-1}$ is a regular sequence. Let

$$
\mu: R \otimes \Lambda\left(Q_{0}, \ldots, Q_{n-1}\right) \rightarrow R \otimes \Lambda^{*}
$$

be an $R$-module isomorphism defined by

$$
\mu\left(Q_{0} \cdots \widehat{Q}_{i_{1}} \cdots \widehat{Q}_{i_{r}} \cdots Q_{n-1}\right)=x_{i_{1}} \cdots x_{i_{r}}
$$

where $0 \leq i_{1}<\cdots<i_{r} \leq n-1$. We verify that this homomorphism $\mu$ commutes with the differentials $d_{1}, d$. On the one hand,

$$
\begin{aligned}
d \circ \mu\left(Q_{0} \cdots \widehat{Q}_{i_{1}} \cdots \widehat{Q}_{i_{r}} \cdots Q_{n-1}\right) & =d\left(x_{i_{1}} \cdots x_{i_{r}}\right) \\
& =\sum_{j=1}^{r}(-1)^{j+1} g_{i_{j}} x_{i_{1}} \cdots \widehat{x_{i_{j}}} \cdots x_{i_{r}} .
\end{aligned}
$$

On the other hand, since

$$
\begin{aligned}
& d_{1}\left(Q_{0} \cdots \widehat{Q}_{i_{1}} \cdots \widehat{Q}_{i_{r}} \cdots Q_{n-1}\right) \\
= & \left(f_{0} Q_{0}+\cdots+f_{n-1} Q_{n-1}\right) Q_{0} \cdots \widehat{Q}_{i_{1}} \cdots \widehat{Q}_{i_{r}} \cdots Q_{n-1} \\
= & (-1)^{i_{1}} f_{i_{1}} Q_{0} \cdots Q_{i_{1}} \cdots \widehat{Q}_{i_{2}} \cdots \widehat{Q}_{i_{r}} \cdots Q_{n-1} \\
& +(-1)^{i_{2}-1} f_{i_{2}} Q_{0} \cdots \widehat{Q}_{i_{1}} \cdots Q_{i_{2}} \cdots \widehat{Q}_{i_{r}} \cdots Q_{n-1} \\
& \vdots \\
& +(-1)^{i_{r}-(r-1)} f_{i_{r}} Q_{0} \cdots \widehat{Q}_{i_{1}} \cdots \widehat{Q}_{i_{2}} \cdots Q_{i_{r}} \cdots Q_{n-1},
\end{aligned}
$$


we get

$$
\begin{aligned}
\mu \circ d_{1}\left(Q_{0} \cdots \widehat{Q}_{i_{1}} \cdots \widehat{Q}_{i_{r}} \cdots Q_{n-1}\right) & =\sum_{j=1}^{r}(-1)^{i_{j}-j+1} f_{i_{j}} x_{i_{1}} \cdots \widehat{x_{i_{j}}} \cdots x_{i_{r}} \\
& =\sum_{j=1}^{r}(-1)^{j+1} g_{i_{j}} x_{i_{1}} \cdots \widehat{x_{i_{j}}} \cdots x_{i_{r}}
\end{aligned}
$$

Therefore, $\mu$ is an isomorphism of chain complexes, so that by Proposition 2.2, Proposition 2.1 holds.

\section{The Dickson invariant and Milnor $Q_{i}$ operation}

Let $A$ be an elementary abelian $p$-group of rank $n$. The $\bmod p$ cohomology $H^{*}(B A)$ is isomorphic to the polynomial tensor exterior algebra

$$
\mathbb{F}_{p}\left[t_{1}, \ldots, t_{n}\right] \otimes \Lambda\left(d t_{1}, \ldots, d t_{n}\right)
$$

as a graded algebra. It is also clear that the finite general linear group $G L\left(n, \mathbb{F}_{p}\right)$ and the finite special linear group $S L\left(n, \mathbb{F}_{p}\right)$ act on $A, B A$ and $H^{*}(B A)$. Dickson computed the ring of invariants of $\mathbb{F}_{p}\left[t_{1}, \ldots, t_{n}\right]$ with respect to the action of $G L\left(n, \mathbb{F}_{p}\right)$. In the case of $G=G L\left(n, \mathbb{F}_{p}\right)$, the ring of invariants is a polynomial algebra

$$
D_{n}=\mathbb{F}_{p}\left[c_{n, 0}, \ldots, c_{n, n-1}\right]
$$

whose generators are given by the equation

$$
\mathcal{O}_{n}(X)=\prod_{t \in \mathbb{F}_{p}\left\{t_{1}, \cdots, t_{n}\right\}}(X+t)=X^{p^{n}}+\sum_{j=0}^{n-1} c_{n, j} X^{p^{j}} .
$$

Remark. In [Ka], [Ka-Mi], notation $c_{n, i}$ is used as $(-1)^{n-i} c_{n, i}$ in this paper.

For the invariant ring under $S L\left(n, \mathbb{F}_{p}\right)$, we have

$$
S D_{n}=D_{n}\left\{1, e_{n}, \ldots, e_{n}^{p-2}\right\} \quad \text { with } e_{n}^{p-1}=c_{n, 0} .
$$

Mui computed the ring of invariants of $\mathbb{F}_{p}\left[t_{1}, \ldots, t_{n}\right] \otimes \Lambda\left(d t_{1}, \ldots, d t_{n}\right)$ with respect to the action of $S L\left(n, \mathbb{F}_{p}\right)$. Of course $u_{n}=d t_{1} \ldots d t_{n}$ is invariant under $S L\left(n, \mathbb{F}_{p}\right)$. In terms of Milnor's operation, we may state Mui's result in the following form.

Theorem 3.1 (Mui [Mu], Kameko-Mimura [Ka-Mi]).

$$
\begin{gathered}
H^{*}(B A)^{S L\left(n, \mathbb{F}_{p}\right)} \cong \mathbb{F}_{p}\left[c_{n, 1}, \ldots, c_{n, n-1}\right] \oplus S D_{n} \otimes \Lambda\left(Q_{0}, \ldots, Q_{n-1}\right)\left\{u_{n}\right\} \\
\cong S D_{n} /\left(e_{n}\right) \oplus S D_{n} \otimes \Lambda\left(Q_{0}, \ldots, Q_{n-1}\right)\left\{u_{n}\right\}
\end{gathered}
$$

where $Q_{0} \ldots Q_{n-1} u_{n}=e_{n}$.

In this section, we compute $f_{0}, \ldots, f_{n-1}$ in $\S 2$ for the case $S=S D_{n}$. We study some important relations between $Q_{i}$ and $c_{n, j}$.

Lemma 3.2. For $x \in H^{*}(B A)$, it follows that

$$
\left(Q_{n}+\sum_{i=1}^{n} c_{n, i} Q_{i}\right)(x)=0, \quad Q_{0} \ldots \hat{Q}_{i} \ldots Q_{n}\left(u_{n}\right)=(-1)^{n-i} c_{n, i} e_{n} .
$$


Proof. Note $Q_{i}\left(d t_{j}\right)=t_{j}^{p^{i}}$. When $x=d t_{j}$, we see

$$
\left(Q_{n}+\sum c_{n, i} Q_{i}\right)\left(d t_{j}\right)=t_{j}^{p^{n}}+\sum c_{n, i} t_{j}^{p^{i}}=\mathcal{O}_{n}\left(t_{j}\right)=0 .
$$

Since $Q_{i}$ is a derivation, we get the first equation.

Applying $Q_{0} \ldots \hat{Q}_{i} \ldots Q_{n-1}$ to the first equation, we get the second equation.

By using the relation $Q_{k+1}=\left[Q_{k}, \wp^{p^{k}}\right]$ and the above lemma, we may define an $\mathcal{E}$-module structure on $S D_{n} \otimes \Lambda\left(Q_{0}, \ldots, Q_{n-1}\right)\left\{u_{n}\right\}$. Let us write (recall the definition in $\S 2$ )

$$
Q_{k} u_{n}=\alpha_{0, k} Q_{0} u_{n}+\cdots+\alpha_{n-1, k} Q_{n-1} u_{n} .
$$

With the following proposition, we verify that there is an algebra homomorphism $\mathcal{E} \rightarrow S D_{n} \otimes \Lambda\left(Q_{0}, \ldots, Q_{n-1}\right)$ such that the evaluation at $u_{n}$ induces an $\mathcal{E}$-module homomorphism $S D_{n} \otimes \Lambda\left(Q_{0}, \ldots, Q_{n-1}\right) \rightarrow H^{*}(B A)$.

Proposition 3.3. For each $x$ in $S D_{n} \otimes \Lambda\left(Q_{0}, \ldots, Q_{n-1}\right)\left\{u_{n}\right\} \subset H^{*} B A$,

$$
Q_{k} x=\left(\alpha_{0, k} Q_{0}+\cdots+\alpha_{n-1, k} Q_{n-1}\right) x .
$$

Proof. It is immediate that $Q_{k} Q_{i_{1}} \cdots Q_{i_{r}}=(-1)^{r} Q_{i_{1}} \cdots Q_{i_{r}} Q_{k}$ and so

$$
\begin{aligned}
Q_{k} Q_{i_{1}} \cdots Q_{i_{r}} u_{n} & =(-1)^{r} Q_{i_{1}} \cdots Q_{i_{r}} Q_{k} u_{n} \\
& =(-1)^{r} Q_{i_{1}} \cdots Q_{i_{r}}\left(\alpha_{0, k} Q_{0} u_{n}+\cdots+\alpha_{n-1, k} Q_{n-1} u_{n}\right) \\
& =\left(\alpha_{k, 0} Q_{0}+\cdots+\alpha_{n-1, k} Q_{n-1}\right) Q_{i_{1}} \cdots Q_{i_{r}} u_{n} .
\end{aligned}
$$

The following propositions are only used in the last section to prove Theorem 1.8 .

Proposition 3.4. For $i \geq 1, \alpha_{i, 0}=0$ and $\alpha_{0,0}=1$. Letting $\alpha_{-1, k}=0$, we have

$$
\alpha_{i, k+1}=\alpha_{i-1, k}^{p}-\alpha_{n-1, k}^{p} c_{n, i} .
$$

Proof. Since $Q_{k+1}=\left[Q_{k}, \wp^{p^{k}}\right]$, we have

$$
Q_{k+1} u_{n}=\wp^{p^{k}} Q_{k} u_{n}=\wp^{p^{k}} \sum_{i=0}^{n-1} \alpha_{i, k} Q_{i} u_{n}=\sum_{i=0}^{n-1} \alpha_{i, k}^{p} Q_{i+1} u_{n} .
$$

The last equation follows from $\operatorname{deg} \alpha_{i, k}=2 p^{k}-2 p^{i}$ and

$$
\wp^{p^{k}} \alpha_{i, k} Q_{i} u_{n}=\wp^{p^{k}-p^{i}} \alpha_{i, k} \cdot \wp^{p^{i}} Q_{i} u_{n}=\alpha_{i, k}^{p} Q_{i+1} u_{n}
$$

We also get

$$
\begin{gathered}
\sum_{i=0}^{n-1} \alpha_{i, k}^{p} Q_{i+1} u_{n}=\sum_{i=0}^{n-2} \alpha_{i, k}^{p} Q_{i+1} u_{n}+\alpha_{n-1, k}^{p} Q_{n} u_{n} \\
=\sum_{i=1}^{n-1} \alpha_{i-1, k}^{p} Q_{i} u_{n}-\sum_{i=0}^{n-1} \alpha_{n-1, k}^{p} c_{n, i} Q_{i} u_{n}
\end{gathered}
$$

Here the last equation follows from Lemma 3.2. Hence the last element is equal to $Q_{k+1} u=\sum \alpha_{i, k+1} Q_{i} u_{n}$. Thus we get the equation in this proposition.

Proposition 3.5. For $k \leq n-1, \alpha_{i, k}=\delta_{i, k}$. 
Proposition 3.6. We have $\alpha_{i, n}=-c_{n, i}$ and $\alpha_{n-1, k} \neq 0$ for $k \geq n$; in particular,

$$
\alpha_{n-1, n+k}=c_{n, n-1}^{p^{k}+\cdots+p+1}+\text { terms lower with respect to } c_{n, n-1} .
$$

In particular, $\alpha_{0, n}=-c_{n, 0}, \ldots, \alpha_{n-1, n}=-c_{n, n-1}, \alpha_{i, j}=\delta_{i, j}$ for $i, j=0, \ldots$, $n-1$. That is, $f_{i}$ is expressed as

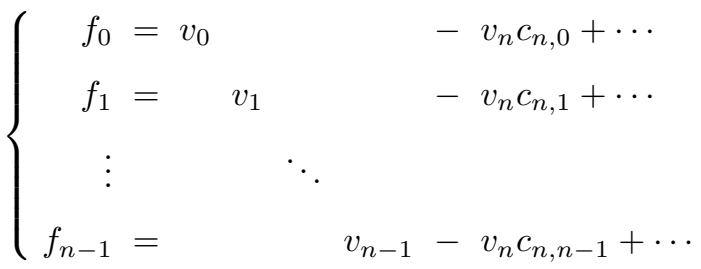

\section{Projective unitary groups $P U(p)$}

The additive structure of the cohomology $H^{*}(B P U(p))$ was first determined by the first author computing the Poincaré series of the $E_{2}$-term of the RothenbergSteenrod spectral sequence. (A. Vistoli [Vi] also gets the same results by completely different methods.) Indeed, we have

$$
\begin{gathered}
P S\left(H^{*}(B P U(p), t)\right)=\sum \operatorname{rank}_{p} H^{i}(B P U(p)) t^{i} \\
=\frac{1}{\left(1-t^{4}\right)\left(1-t^{6}\right) \ldots\left(1-t^{2 p}\right)}+\frac{t^{2}+t^{3}+t^{2 p+1}+t^{2 p+2}}{\left(1-t^{2 p+2}\right)\left(1-t^{2 p(p-1)}\right)} .
\end{gathered}
$$

However we give it here by a short argument using the results of Vavpetič and Viruel [Va-Vi] and Kono and Yagita [Ko-Ya].

Let $G$ be a compact Lie group. Quillen showed that the following restriction map $r$ is an F-isomorphism:

$$
r: H^{*}(B G) \rightarrow \underset{\leftarrow}{\lim } H^{*}(B A)
$$

where $A$ runs over a set of conjugacy classes of elementary abelian $p$-subgroups of $G$. Here an F-isomorphism means that its kernel is generated by nilpotent elements and for each $x \in H^{*}(B G)$ there is $s \geq 0$ such that $x^{p^{s}} \in \operatorname{Im}(r)$.

Recently A.Vavpetič and A.Viruel gave the following short proof of the fact that the map $r$ is injective for $G=P U(p)$.

There is only one conjugacy class of nontoral abelian groups $A$ of $\operatorname{rank}_{p}=2$. Let $T_{G}$ be a maximal torus of $G$ and $N_{G}=N_{G}(T)$ the normalizer of the torus $T_{G}$. Let $N_{p}(G)$ be the $p$-normalizer of $T_{G}$, namely, the preimage of a $p$-Sylow subgroup in the Weyl group $W_{G}=N_{G} / T_{G}$. For example, it is known that

$$
N_{p}(U(p)) \cong S^{1} \imath \mathbb{Z} / p \cong\left\{\operatorname{diag}\left(z_{1}, \ldots, z_{p}\right) P^{i} \in U(p) \mid z_{j} \in \mathbb{C}^{*}, 0 \leq i \leq p-1\right\}
$$

where $P \in U(p)$ is a permutation matrix of order $p$.

Lemma 4.1. $N_{p}(S U(p)) \cong N_{p}(P U(p))$.

The cofiber sequence $\left(\mathbb{C}^{*} \cong\right) S^{1} \rightarrow B S U(p) \rightarrow B U(p)$ induces the Gysin exact sequence

$$
\rightarrow H^{*-2}\left(B N_{p}(U(p))\right) \rightarrow H^{*}\left(B N_{p}(U(p))\right) \rightarrow H^{*}\left(B N_{p}(S U(p))\right) \rightarrow .
$$


Considering restrictions to $A$ and tori of $U(p)$ and $S U(p)$, Vavpetič and Viruel prove :

Lemma $4.2([\mathrm{Va}-\mathrm{Vi}]) \cdot H^{*}\left(B N_{p}(S U(p))\right)$ is detected by $A$ and $T_{S U(p)}$.

Theorem $4.3([\mathrm{Va}-\mathrm{Vi}]) \cdot H^{*}(B P U(p))$ is detected by $A$ and $T_{P U(p)}$.

Proof. Consider the restrictions

$$
H^{*}(B P U(p)) \stackrel{i_{N_{P U}}}{\rightarrow} H^{*}\left(B N_{P U(p)}\right) \stackrel{i_{N_{p}}}{\rightarrow} H^{*}\left(B N_{p}(P U(p))\right) \cong H^{*}\left(B N_{p}(S U(p))\right) .
$$

Recall the transfer $t r$ and Gottlieb transfer Gtr so that Gtr. $i_{N_{P U}}=i d$ and $t r . i_{N_{p}}=$ $i d$. Hence the above restrictions are injective.

Note that $W_{G}(A)=S L\left(2, \mathbb{F}_{p}\right)$ for $G=P U(p)$. Next by using arguments ([Ko-Ya]), we will show for $G=P U(p)$ that the following map $p i_{A}$ is epic:

$$
\begin{aligned}
p i_{A}: H^{*}(B G) \stackrel{i_{A}}{\rightarrow} H^{*}(B A)^{W_{G}(A)}=S D_{2} /\left(e_{2}\right) \oplus S D_{2} \otimes \Lambda\left(Q_{0}, Q_{1}\right)\left\{u_{2}\right\} \\
\stackrel{\text { proj. }}{\longrightarrow} S D_{2} \otimes \Lambda\left(Q_{0}, Q_{1}\right)\left\{u_{2}\right\} .
\end{aligned}
$$

Let $E$ be the subgroup of $S U(p)$ generated by

$$
c=\operatorname{diag}(\xi, \ldots, \xi), \quad a=\operatorname{diag}\left(1, \xi, \ldots, \xi^{p-1}\right), \quad b=P \text { (permutation matrix) }
$$

where $\xi$ is the $p$-th primitive root of 1 . Then we see that

$$
a^{p}=b^{p}=c^{p}=1, \quad[a, b]=c \in \text { center of } E .
$$

Hence $E$ is isomorphic to the extraspecial $p$-group $p_{+}^{1+2}$ of order $p^{3}$. We consider the commutative diagram for fiberings

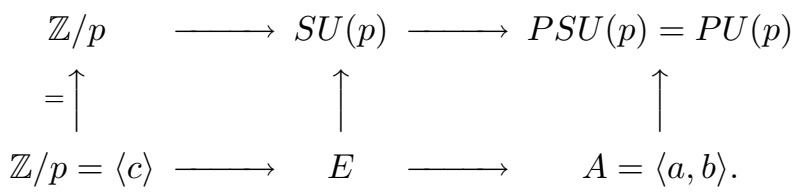

The above diagram induces the map $f_{r}: E(U)_{r}^{*, *} \rightarrow E(E)_{r}^{*, *}$ from the spectral sequence converging to $H^{*}(B S U(p))$ to that converging to $H^{*}(B E)$.

The differential of $E(E)_{r}^{*, *}$ is well known [Te-Ya]. Let us write

$$
E(E)_{2}^{*, *} \cong H^{*}\left(B A ; H^{*}(B \mathbb{Z} / p)\right) \cong \mathbb{F}_{p}\left[t_{1}, t_{2}, w\right] \otimes \Lambda\left(d t_{1}, d t_{2}, d w\right)
$$

with $\beta d w=w$. Then it is known that

$$
d_{2}(d w)=d t_{1} d t_{2}=u_{2} .
$$

Since $d w \in \operatorname{Im}\left(f_{2}\right)$, we see that $u_{2} \in \operatorname{Im}\left(f_{3}\right)$ and so in $\operatorname{Im}\left(p i_{A}^{*}\right)$.

Here we also see that $c_{2,1}, c_{2,0} \in \operatorname{Im}\left(i_{A}^{*}\right)$. Let $\rho$ be the canonical representation of $S U(p)$ and

$$
\lambda=\rho \otimes \rho^{-1}: S U(p) \rightarrow S U\left(p^{2}\right) .
$$

Since $\rho \otimes \rho^{-1} \mid \mathbb{Z} / p$ is trivial, we can identify $\lambda$ as the representation of $P U(p)$. For $a^{i} b^{j} \in A \cong(\mathbb{Z} / p)^{2}$, we easily compute $\chi_{\lambda}\left(a^{i} b^{j}\right)=p^{2}$ if $i=j=0$ and $=0$ otherwise. This means that $\lambda \mid A$ is the regular representation. So the total Chern class is

$$
c(\lambda \mid A)=\Pi_{\left(\lambda_{1}, \lambda_{2}\right) \in(\mathbb{Z} / p)^{2}}\left(1+\lambda_{1} t_{1}+\lambda_{2} t_{2}\right)=1+c_{2,1}+c_{2,0} .
$$

Hence we have $c_{p^{2}-p}(\lambda) \mid A=c_{2,1}$ and $c_{p^{2}-1}(\lambda) \mid A=c_{2,0}$. 
Lemma 4.4. The map $p i_{A}$ is epic.

(From Lemma 3.2, we also have $e_{2}=Q_{0} Q_{1} u_{2}, e_{2}^{p-1}=c_{2,0}$ and $Q_{2} Q_{0}\left(u_{2}\right)=$ $\left.c_{2,1} e_{2}.\right)$

Thus we get the short exact sequence

$$
0 \rightarrow \operatorname{Ker}\left(p i_{A}\right) \rightarrow H^{*}(B P U(p)) \rightarrow S D_{2} \otimes \Lambda\left(Q_{0}, Q_{1}\right)\left\{u_{2}\right\} \rightarrow 0 .
$$

By the theorem by Vavpetič and Viruel, we have the injection

$$
i_{T} \times i_{A}: \operatorname{Ker}\left(p i_{A}\right) \subset H^{*}(B P U(p)) \rightarrow H^{*}\left(B T_{P U(p)}\right) \times S D_{2} /\left(e_{2}\right) .
$$

In particular $\operatorname{Ker}\left(p i_{A}\right)$ is generated by even-dimensional elements.

Now we consider the Bockstein spectral sequence

$$
E_{1}=H^{*}(X) \Longrightarrow \mathbb{F}_{p} \otimes H^{*}(X ; \mathbb{Z}) /(\text { tor sions }) .
$$

The first differential is the Bockstein operation $Q_{0}$. Since $\operatorname{Im}\left(p i_{A}\right)$ is $Q_{0}$-free, so $H\left(\operatorname{Im}\left(p i_{A}\right) ; Q_{0}\right)=0$. Hence we have

$$
E_{2}=H\left(H^{*}\left(B P U(p) ; Q_{0}\right)\right) \cong \operatorname{Ker}\left(p i_{A}\right) .
$$

Since $\operatorname{Ker}\left(p i_{A}\right)$ is generated by even-dimensional elements, we get

$$
E_{\infty} \cong E_{2} \cong \operatorname{Ker}\left(p i_{A}\right)
$$

It is well known that

$$
H^{*}(B P U(p) ; \mathbb{Q}) \cong H^{*}(B S U(p) ; \mathbb{Q}) \cong \mathbb{Q}\left[c_{2}, \ldots, c_{p}\right] .
$$

Since $\operatorname{rank}_{p}\left(\mathbb{F}_{p} \otimes\left(H^{i}(X ; \mathbb{Z}) /(\right.\right.$ torsions $\left.\left.)\right)\right)=\operatorname{rank}_{\mathbb{Q}} H^{i}(X ; \mathbb{Q})$, we get

Lemma 4.5. There exist additive isomorphisms

$$
\operatorname{Ker}\left(p i_{A}\right) \cong \mathbb{F}_{p} \otimes H^{*}(B P U(p) ; \mathbb{Z}) /(\text { torsions }) \cong \mathbb{F}_{p}\left[x_{2}, \ldots, x_{p}\right] \text { with }\left|x_{i}\right|=2 i .
$$

Remark. This is not a ring isomorphism; e.g., for $p=3$ we have the ring isomorphism

$$
\operatorname{Ker}\left(p i_{A}\right) \cong \mathbb{F}_{3}\left[c_{2}, c_{3}, c_{6}\right] /\left(c_{2}^{3}-c_{3}^{2}\right) ¥ \mathbb{F}_{3}\left[c_{2}, c_{3}\right] .
$$

Thus we have Theorem 1.7 in the introduction.

Theorem 4.6. There exists a short exact sequence of rings

$$
0 \rightarrow M / p \rightarrow H^{*}(B P U(p)) \rightarrow S D_{2} \otimes \Lambda\left(Q_{0}, Q_{1}\right)\left\{u_{2}\right\} \rightarrow 0
$$

with additively (not rings) $M / p \cong \mathbb{F}_{p}\left[x_{2}, \ldots, x_{p}\right]$.

Now we consider the Adams spectral sequence converging to $P(m)^{*}(B P U(p))$. By the change of rings,

$$
\operatorname{Ext}_{A}^{s, t}\left(H^{*}\left(P(m), H^{*}(X)\right) \cong \operatorname{Ext}_{\mathcal{E}_{m}}^{s, t}\left(\mathbb{F}_{p}, H^{*}(X)\right) .\right.
$$

From the above theorem, Theorem 1.8 implies

Lemma 4.7. There exists a short exact sequence

$$
0 \rightarrow P(m)^{*} \hat{\otimes} M \rightarrow \operatorname{Ext}_{\mathcal{E}_{m}}\left(\mathbb{F}_{p}, H^{*}(B P U(p)) \rightarrow P(m)^{*} \hat{\otimes} S D_{2} /\left(f_{0}, f_{1}\right)\left\{e_{2}\right\} \rightarrow 0\right.
$$


Thus we have Theorem 1.1 in the introduction for $G=P U(p)$.

Next we consider the Chow ring for $B P U(p)$. Recall that $N_{p}(U(p)) \cong S^{1} \imath \mathbb{Z} / p$. By Totaro, it is known that $C H^{*}\left(S^{1} \prec \mathbb{Z} / p\right) / p$ is detected by $C H^{*}\left(\left(B S^{1}\right)^{\times p}\right) / p$ and $C H^{*}\left(B\left(S^{1} \times \mathbb{Z} / p\right)\right) / p$ (see page 271 in [To]) and so by elementary $p$-subgroups. We also know that the restriction map

$$
C H^{*}\left(B\left(S^{1} \curlywedge \mathbb{Z} / p\right)\right) \rightarrow C H^{*}\left(\left(B S^{1}\right)^{\times p}\right)^{\mathbb{Z} / p} / p \cong \mathbb{Z} / p\left[y_{1}, \ldots, y_{p}\right]^{\mathbb{Z} / p}
$$

is epic. Moreover he showed (his conjecture)

$$
C H^{*}(B G) \cong B P^{*}(B G) \otimes_{B P^{*}} \mathbb{Z}_{(p)}
$$

for this group $G=S^{1} \imath \mathbb{Z} / p$. By using $C H^{*}(-) / p$ (and $B P^{*}(-)$ ) version of the Gysin sequence given just before Lemma 4.2 , we also see $(*)$ for $G=N_{p}(S U(p))$. Since Chow rings $C H^{*}(-)_{(p)}$ (and $\left.B P^{*}(-) \otimes_{B P^{*}} \mathbb{Z}_{(p)}\right)$ have transfer (and Gottlieb transfer), the isomorphism $(*)$ for $G=P U(p)$ follows from the arguments of the proof of Theorem 4.3. Recall $P G L(p, \mathbb{C})$ is the algebraic group corresponding to $P U(p)$.

Theorem 4.8. $C H^{*}(B P G L(p, \mathbb{C}))_{(p)} \cong B P^{*}(B P U(p)) \otimes_{B P^{*}} \mathbb{Z}_{(p)}$.

Thus we get Theorem 1.5 in the introduction.

Remark. Vezzosi first studied $C H^{*}(B P G L(3, \mathbb{C}))_{(3)}=C H^{*}(P U(3))_{(3)}$. He showed (Theorem 1.1 in [Ve]) if some element $\chi \in C H^{6}(P U(3))_{(3)}$ is zero, then we have the isomorphism in the above theorem. Indeed, we have seen $\chi=0$.

Let $H^{*, *}(-; \mathbb{Z})$ be the motivic cohomology defined by Suslin and Voevodsky so that $H^{2 *, *}(X ; \mathbb{Z}) \cong C H^{*}(X)$. Here $H^{2 *, *}(X ; \mathbb{Z})=\bigoplus_{i} H^{2 i, i}(X ; \mathbb{Z})$. Recall that $M G L^{*, *}(-)$ is the motivic cohomology defined by Voevodsky ([Vo1]) by using spectrum $M G L$ in the $\mathbb{A}^{1}$ stable homotopy category. Of course there is the natural $\operatorname{map} M G L^{2 *, *}(X) \rightarrow M U^{2 *}(X)$.

Corollary 4.9. $M G L^{2 * * *}(B G L(p, \mathbb{C}))_{(p)} \cong M U^{2 *}(B P U(p))_{(p)}$.

Proof. Let $X=B P G L(p, \mathbb{C})$. Consider the motivic Atiyah-Hirzebruch spectral sequence

$$
E_{2}=H^{*, *}\left(X ; M U^{*}\right) \Longrightarrow M G L^{*, *}(X) .
$$

When $X$ is smooth and $m>2 n$, it is known that $H^{m, n}(X ; \mathbb{Z})=0$. This implies $((1.2)$ in $[\mathrm{Ya}])$

$$
M G L^{2 *, *}(X) \otimes_{M U^{*}} \mathbb{Z} \cong H^{2 *, *}(X ; \mathbb{Z}) \cong C H^{*}(X) .
$$

From the above theorem, we only need to prove that there is a relation such that

$$
f_{1} x_{2 p+2}=v_{1} e_{2}+\ldots=0 \quad \text { also in } M G L^{2 *, *}(X)
$$

$\left(x_{2 p+2}=e_{2}\right.$ in the notation of Theorem 1.4 and Theorem 4.6). By the solution of the Bloch-Kato conjecture of degree 2 by Merkurjev-Suslin, we get

$$
u_{2} \in H^{2,2}(X ; \mathbb{Z} / p) \text { and } Q_{0}\left(u_{2}\right) \in H^{3,2}\left(X ; \mathbb{Z}_{(p)}\right),
$$

and hence $e=Q_{1} Q_{0}\left(u_{2}\right) \in H^{2 p+2, p+1}\left(X ; \mathbb{Z}_{(p)}\right)=C H^{p+1}(X)_{(p)}$. The first differential of the Atiyah-Hirzebruch spectral sequence is given by ((4.6) in [Ya])

$$
d_{p-1}\left(Q_{0} u_{2}\right)=v_{1} \otimes Q_{1} Q_{0}\left(u_{2}\right)=v_{1} \otimes e_{2} .
$$

Hence $v_{1} e_{2}=0$ in $\operatorname{gr} M G L^{2 *, *}(X)$. 


\section{The eXCeptional Lie groups CASES}

With the result on Poincaré series of cotorsion products, the first author proves that the Rothenberg-Steenrod spectral sequence for the mod $p$ cohomology of $B G$ collapses at the $E_{2}$-level and the Quillen homomorphism $r$ is a monomorphism in the cases $G=E_{8}$ and $p=5, G=F_{4}, E_{6}, E_{7}$ and $p=3$. In these cases, each exceptional Lie group has two conjugacy classes of maximal elementary abelian $p$-groups. One is the subgroup of a maximal torus and the other is a nontoral $A$. Let us write $i_{A}: B A \rightarrow B G$ and $i_{T}: B T_{G} \rightarrow B G$ for the induced maps from the inclusions. (However note that for $G=E_{8}$ and $p=3$, there are two conjugacy classes of nontoral maximal elementary abelian 3-subgroups.)

Theorem 5.1 ([Ka]). In the case $G=E_{8}$ and $p=5, G=F_{4}, E_{6}, E_{7}$ and $p=$ 3 , the induced homomorphism $i_{A}^{*} \times i_{T}^{*}: H^{*}(B G) \rightarrow H^{*}(B A) \times H^{*}\left(B T_{G}\right)$ is a monomorphism.

Moreover $\operatorname{Im}\left(i_{A}^{*}\right)$ is also known for these groups.

Case I. Consider the cases $G=E_{8}$ and $p=5, G=F_{4}$ and $p=3$. In these cases $W_{G}(A)=S L\left(3, \mathbb{F}_{p}\right)$. Let

$$
p i_{A}: H^{*}(B G) \rightarrow H^{*}(B A)^{W_{G}(A)} \rightarrow S D_{3} \otimes \Lambda\left(Q_{0}, Q_{1}, Q_{2}\right)\left\{u_{3}\right\} .
$$

Let $K=\operatorname{Ker}\left(p i_{A}\right)$ and $I M=\operatorname{Im}\left(p i_{A}\right)$. The map $p i_{A}$ is not epic, indeed $u_{3} \notin I M$, and we can show

Lemma 5.2. There exists a short exact sequence

$$
0 \rightarrow I M \rightarrow S D_{3} \otimes \Lambda\left(Q_{0}, Q_{1}, Q_{2}\right)\left\{u_{3}\right\} \rightarrow \mathbb{F}_{p}\left\{u_{3}\right\} \rightarrow 0 .
$$

Thus we get

$$
\operatorname{Ext}_{\mathcal{E}_{m}}\left(\mathbb{F}_{p}, I M\right) \cong P(m)^{*} \hat{\otimes} S D_{3} /\left(f_{0}, f_{1}, f_{2}\right)\left\{e_{3}\right\} \oplus P(m)^{*}\left\{\delta u_{3}\right\}, \quad\left|\delta u_{3}\right|=4 .
$$

Since $K=\operatorname{Ker}\left(p i_{A}\right)$ is a trivial $\mathcal{E}_{m}$-module generated by even-dimensional elements, we get Theorem 1.1 for these groups.

Proposition 5.3. For $G$ in case $I, \operatorname{Ext}_{\mathcal{E}_{m}}\left(\mathbb{F}_{p}, H^{*}(B G)\right)$ is isomorphic to

$$
P(m)^{*} \hat{\otimes} S D_{3} /\left(f_{0}, f_{1}, f_{2}\right)\left\{e_{3}\right\} \oplus P(m)^{*}\left\{\delta u_{3}\right\} \oplus P(m)^{*} \hat{\otimes} K .
$$

Next consider the Bockstein spectral sequence

$$
E_{1}=H^{*}(B G) \Longrightarrow \mathbb{F}_{p} \otimes H^{*}(B G ; \mathbb{Z}) /(\text { tor sions }) .
$$

Note $I M$ is not $\Lambda\left(Q_{0}\right)$-free but

$$
E_{2}=H\left(H^{*}(B G) ; Q_{0}\right) \cong K \oplus \mathbb{F}_{p}\left\{Q_{0} u_{3}\right\} .
$$

Hence the Poincaré series $P S_{R}(-, t)$ (for graded $R$-vector spaces) is given by

$$
\begin{aligned}
& P S_{\mathbb{Z} / p}\left(K \oplus \mathbb{Z} / p\left\{\delta_{3}\right\}, t\right)=P S_{\mathbb{Z} / p}(K, t)+t^{4} \\
& \quad=P S_{\mathbb{Z} / p}\left(K \oplus \mathbb{Z} / p\left\{Q_{0} u_{3}\right\}, t\right)=P S_{\mathbb{Q}}\left(H^{*}(B G ; \mathbb{Q}), t\right) .
\end{aligned}
$$

Thus we get

Theorem 5.4. For the case $I$, there exists a $P(m)^{*}$-algebra exact sequence

$$
0 \rightarrow P(m)^{*} \hat{\otimes} M \rightarrow \operatorname{gr} P(m)^{*}(B G) \rightarrow P(m)^{*} \hat{\otimes} S D_{3} /\left(f_{0}, f_{1}, f_{3}\right)\left\{e_{3}\right\} \rightarrow 0 .
$$


(1) $M \cong \mathbb{Z}_{(3)}\left[x_{4}, x_{12}, x_{16}, x_{24}\right]$ as $\mathbb{Z}_{(3)}$-modules for $G=F_{4}, p=3$.

(2) $M \cong \mathbb{Z}_{(5)}\left[x_{4}, x_{16}, x_{24}, x_{28}, x_{36}, x_{40}, x_{48}, x_{60}\right]$ as $\mathbb{Z}_{(5)}$-modules for $G=E_{8}$, $p=5$.

The mod 3 cohomology of $B F_{4}$ is completely determined by Toda.

Theorem 5.5 ([Toda]). The cohomology $H^{*}\left(B F_{4}\right)$ is isomorphic to $\mathbb{Z} / 3\left[x_{36}, x_{48}\right] \otimes\left(\mathbb{Z} / 3\left[x_{4}, x_{8}\right] \otimes\left\{1, x_{20}, x_{20}^{2}\right\}+\mathbb{Z} / 3\left[x_{26}\right] \otimes \Lambda\left(x_{9}\right) \otimes\left\{1, x_{20}, x_{21}, x_{25}\right\}\right)$ where the above two terms have the intersection $\left\{1, x_{20}\right\}$.

Indeed, we see that $x_{26}\left|A=e_{3}, x_{36}\right| A=c_{3,1}, x_{48}\left|A=c_{3,2}, x_{4}\right| A=Q_{0}\left(u_{3}\right), x_{8} \mid A$ $=Q_{1}\left(u_{3}\right), x_{20}\left|A=Q_{2}\left(u_{3}\right), x_{9}\right| A=Q_{0} Q_{1}\left(u_{3}\right), x_{21}\left|A=Q_{0} Q_{2}\left(u_{3}\right), x_{25}\right| A=$ $Q_{1} Q_{2}\left(u_{3}\right)$. The Brown-Peterson theory $B P^{*}(B G)$ is also computed in [Ko-Ya] by using the Atiyah-Hirzebruch spectral sequence. The ring structure of $M$ in Theorem 5.4 is quite complicated

Proposition $5.6([\mathrm{Ko}-\mathrm{Ya}]) \cdot M \cong D_{3} /\left(c_{3,0}\right) \otimes\left(\mathbb{Z}_{(3)}\left\{1,3 x_{4}\right\} \oplus E\right)$ with

$$
E=\mathbb{Z}_{(3)}\left[x_{4}, x_{8}\right]\left\{a b \mid a, b \in\left\{x_{4}, x_{8}, x_{20}\right\}\right\} \subset \mathbb{Z}_{(3)}\left[x_{4}, x_{8}\right]\left\{1, x_{20}, x_{20}^{2}\right\} .
$$

The Poincaré series of $\left(\mathbb{Z} / 3\left\{1,3 x_{4}\right\} \oplus E / 3\right)$ is $a /\left(1-t^{4}\right)\left(1-t^{8}\right)$ with

$$
\begin{gathered}
a=1+t^{20}+t^{40}-\left(t^{8}+t^{20}\right)\left(1-t^{4}\right)\left(1-t^{8}\right) \\
=1-t^{8}+t^{12}+t^{16}-t^{20}+t^{24}+t^{28}-t^{32}+t^{40}=\left(1-t^{8}+t^{16}\right)\left(1+t^{12}+t^{24}\right) .
\end{gathered}
$$

Then we have

$$
\begin{gathered}
P_{\mathbb{Z} / 3}\left(D_{3} /\left(c_{3,0}\right) \otimes\left(\mathbb{Z} / 3\left\{1,3 x_{4}\right\} \oplus E\right), t\right)=\frac{1}{\left(1-t^{36}\right)\left(1-t^{48}\right)} \times \frac{a}{\left(1-t^{4}\right)\left(1-t^{8}\right)} \\
=\frac{a\left(1+t^{8}\right)}{\left(1-t^{12}\right)\left(1+t^{12}+t^{24}\right)\left(1-t^{24}\right)\left(1+t^{24}\right)\left(1-t^{4}\right)\left(1-t^{16}\right)} .
\end{gathered}
$$

Here we see that

$$
\left(1+t^{8}\right) a=\left(1+t^{8}\right)\left(1-t^{8}+t^{16}\right)\left(1+t^{12}+t^{24}\right)=\left(1+t^{24}\right)\left(1+t^{12}+t^{24}\right) .
$$

Hence the above Poincaré series is indeed equal to

$$
P_{\mathbb{Z} / 3}(M / 3, t)=1 /\left(1-t^{12}\right)\left(1-t^{24}\right)\left(1-t^{4}\right)\left(1-t^{16}\right) .
$$

Proposition 5.7. Let $c l: C H^{*}(X) / p \rightarrow H^{*}(X)$ be the mod $p$ cycle map. For $G$ of Case I, we have $\mathrm{pi}_{A} \cdot \operatorname{cl}\left(C H^{*}(B G)\right)=S D_{3}\left\{e_{3}\right\}$.

Proof. From Lemma 9.6 in [Ya] and the affirmative answer to the Bloch-Kato conjecture, there is an element $x \in H^{4,3}(B G ; \mathbb{Z} / p)$ with $\operatorname{cl}(x)=Q_{0} e_{3}$. Here note

$$
Q_{i} Q_{j}(x) \in H^{2 p^{i}+2 p^{j}+2, p^{i}+p^{j}+1}(B G ; \mathbb{Z} / p)=C H^{p^{i}+p^{j}+1}(B G) / p .
$$

Thus $e_{3}, c_{3,1} e_{3}, c_{3,2} e_{3}$ are in the image of the cycle map.

Case II. The groups $G$ are $E_{6}$ and $E_{7}$ for $p=3$.

For each of these cases, $G$ contains a maximal nontoral elementary abelian 3subgroup $A \cong(\mathbb{Z} / 3)^{4}$. It is known (e.g., [A-G-M-V]) that $W_{G}(A)$ is the subgroup 
of $S L\left(4, \mathbb{F}_{3}\right)$ generated by matrices of the form

$$
\left(\begin{array}{cccc} 
& & & 0 \\
& S L\left(3, \mathbb{F}_{3}\right) & & 0 \\
& & & 0 \\
* & * & * & \epsilon
\end{array}\right)
$$

where $* \in \mathbb{F}_{3}, \epsilon=1$ (resp. $\epsilon= \pm 1$ ) for $G=E_{6}$ (resp. $G=E_{7}$ ).

Recall that

$$
\mathcal{O}_{3}(X)=\Pi_{\left(\lambda_{1}, \lambda_{2}, \lambda_{3}\right) \in(\mathbb{Z} / 3)^{3}}\left(X+\lambda_{1} t_{1}+\lambda_{2} t_{2}+\lambda_{3} t_{3}\right) .
$$

Let us denote by $\mathcal{O}$ simply $\mathcal{O}_{3}\left(t_{4}\right)$ so that $e_{4}=Q_{0} Q_{1} Q_{2} Q_{3}\left(u_{4}\right)=e_{3} \mathcal{O}$. Then the invariant ring is computed by Kameko and Mimura [Ka-Mi]:

$H^{*}(B A)^{W_{G}(A)} \cong\left\{\begin{array}{l}S D_{3} /\left(e_{3}\right)[\mathcal{O}] \oplus S D_{3}[\mathcal{O}] \otimes \Lambda\left(Q_{0}, Q_{1}, Q_{2}\right)\left\{u_{3}, u_{4}\right\} \text { for } G=E_{6} \\ S D_{3} /\left(e_{3}\right)\left[\mathcal{O}^{2}\right] \oplus S D_{3}\left[\mathcal{O}^{2}\right] \otimes \Lambda\left(Q_{0}, Q_{1}, Q_{2}\right)\left\{u_{3}, \mathcal{O} u_{4}\right\} \text { for } G=E_{7} .\end{array}\right.$

At first consider the case $G=E_{6}$. Let us write $N=S D_{3}[\mathcal{O}] \otimes \Lambda\left(Q_{0}, Q_{1}, Q_{2}\right)\left\{u_{3}, u_{4}\right\}$ and let the projection be

$$
p i_{A}: H^{*}(B G) \rightarrow H^{*}(B A)^{W_{G}(A)} \rightarrow N .
$$

Lemma 5.8. $\mathcal{O} u_{3}=\left(Q_{3}+c_{3,2} Q_{2}+c_{3,1} Q_{1}+c_{3,0} Q_{0}\right)\left(u_{4}\right)$.

Proof. From Lemma 3.2, we have

$$
\left(Q_{3}+\sum c_{3, i} Q_{i}\right)\left(u_{4}\right)=\left(Q_{3}+\sum c_{3, i} Q_{i}\right)\left(d t_{4}\right) \times d t_{1} d t_{2} d t_{3}=\mathcal{O}\left(t_{4}\right) u_{3} .
$$

Hence we have the decomposition

$$
N=S D_{3} \otimes \Lambda\left(Q_{0}, Q_{1}, Q_{2}\right)\left\{u_{3}\right\} \oplus S D_{3}[\mathcal{O}] \otimes \Lambda\left(Q_{0}, Q_{1}, Q_{2}, Q_{3}\right)\left\{u_{4}\right\} .
$$

We can see that $f_{0}, \ldots, f_{3}$ is not regular in $S D_{3}[\mathcal{O}]$ (while it is regular in $S D_{4}$ ) for general $m \geq 1$. Hence we assume here $m=0$. Take $\operatorname{Ext}_{\mathcal{E}}\left(\mathbb{F}_{p},-\right)$.

Lemma 5.9. The module $\operatorname{Ext}_{\mathcal{E}}\left(\mathbb{F}_{p}, N\right)$ is isomorphic to

$$
B P^{*} \hat{\otimes} S D_{3}\left\{e_{3}\right\} /\left(v_{1}, v_{2}\right) \oplus B P^{*} \hat{\otimes} S D_{3}[\mathcal{O}]\left\{e_{3} \mathcal{O}\right\} /\left(v_{1}, v_{2}, v_{3}\right) \text {. }
$$

It is known that the image $I M=\operatorname{Im}\left(p i_{A}\right)$ is an algebra over $\mathcal{A}$ generated by $Q_{0} Q_{1} u_{4}, Q_{0} u_{3}$ and $S D_{3}, \mathcal{O}$; namely,

$$
N / I M \cong S D_{3}[\mathcal{O}]\left\{u_{3}, u_{4}, Q_{0} u_{4}, Q_{1} u_{4}, Q_{2} u_{4}, Q_{3} u_{4}\right\} .
$$

The above fact is also known in Kono-Mimura [Ko-Mi]; indeed, $H^{*}\left(B E_{6}\right)$ is still computed in [Ko-Mi].

Lemma 5.10. $\operatorname{Ext}_{\mathcal{E}}\left(\mathbb{F}_{p}, N / I M\right)$ is isomorphic to

$$
P(0)^{*} \hat{\otimes} S D_{3}[\mathcal{O}]\left\{u_{3}, Q_{0} u_{4}, Q_{1} u_{4}, Q_{2} u_{4}, Q_{3} u_{4}\right\} /\left(3 Q_{0} u_{4}\right),
$$

which is generated by odd-dimensional elements where $P(0)^{*}=\mathbb{Z} / 3\left[3, v_{1}, \ldots\right]$.

From the above lemmas, we know that $\operatorname{Ext}_{\mathcal{E}}\left(\mathbb{F}_{p}, \operatorname{Im}\left(p i_{A}\right)\right)$ is generated by evendimensional elements. Of course, so is $\operatorname{Ext}_{\mathcal{E}}\left(\mathbb{F}_{p}, \operatorname{Ker}\left(p i_{A}\right)\right)$, and hence we have

Theorem 5.11. For $\left(E_{6}, p=3\right)$, there exists a short exact sequence

$$
0 \rightarrow B P^{*} \hat{\otimes} M \rightarrow \operatorname{grBP} P^{*}\left(B E_{6}\right) \rightarrow I N \rightarrow 0 .
$$

(1) $I N \cong B P^{*} \hat{\otimes} S D_{3}[\mathcal{O}]\left\{\delta Q_{0} u_{4}\right\} \oplus B P^{*} \hat{\otimes} S D_{3}\left\{e_{3}\right\} /\left(v_{1}, v_{2}\right) \oplus B P^{*} \hat{\otimes}$ $S D_{3}[\mathcal{O}]\left\{e_{3} \mathcal{O}\right\} /\left(v_{1}, v_{2}, v_{3}\right)$.

(2) $M \cong \mathbb{Z}_{(3)}\left[x_{4}, x_{10}, x_{12}, x_{16}, x_{18}, x_{24}\right]$ as additively. 
Next consider the case $\left(G=E_{7}, p=3\right)$. It is known that

$$
i_{A}\left(H^{*}(B G)\right) \subset S D_{3} /\left(e_{3}\right)\left[\mathcal{O}^{2}\right] \oplus S D_{3}\left[\mathcal{O}^{2}\right] \otimes \Lambda\left(Q_{0}, Q_{1}, Q_{2}\right)\left\{u_{3}\right\}
$$

(but not in the submodule $\left.S D_{3}[\mathcal{O}] \otimes \Lambda\left(Q_{0}, Q_{1}, Q_{2}\right)\left\{\mathcal{O} u_{4}\right\}\right)$. Let us write $N=$ $S D_{3}\left[\mathcal{O}^{2}\right] \otimes \Lambda\left(Q_{0}, Q_{1}, Q_{2}\right)\left\{u_{3}\right\}$ and let the projection

$$
p i_{A}: H^{*}(B G) \rightarrow H^{*}(B A)^{W_{G}(A)} \rightarrow N \text {. }
$$

We also know that

$$
N / \operatorname{Im}\left(p i_{A}\right) \cong S D_{3}\left[\mathcal{O}^{2}\right]\left\{u_{3}\right\} .
$$

Hence we get Theorem 1.1 by an argument similar to (but more easily than) the case $E_{6}$.

Theorem 5.12. For $\left(G=E_{7}, p=3\right)$, there exists a short exact sequence

$$
0 \rightarrow P(m)^{*} \hat{\otimes} M \rightarrow \operatorname{gr} P(m)^{*}\left(B E_{7}\right) \rightarrow P(m)^{*} \hat{\otimes} S D_{3}\left[\mathcal{O}^{2}\right]\left\{e_{3}\right\} /\left(f_{0}, f_{1}, f_{2}\right) \rightarrow 0
$$

where $M \cong \mathbb{Z}_{(3)}\left[x_{4}, x_{12}, x_{16}, x_{20}, x_{24}, x_{28}, x_{36}\right]$ as additively.

\section{Proof of Theorem 1.8}

For the sake of notational ease, we write $R_{m, n}$ for $P(m)^{*} \widehat{\otimes} S D_{n}$ and $J_{\ell}$ for the ideal $\left(f_{\ell}, \ldots, f_{n-1}\right) \subset P(m)^{*} \widehat{\otimes} D_{n}$.

Proof of Theorem 1.8 in the case $m=0$. It is clear that $f_{i}=v_{i}-v_{n} c_{n, i}+\cdots$ for $i=0, \ldots, n-1$. The above $f_{0}, \ldots, f_{n-1}$ is a regular sequence in $R_{0, n}$. Let $\rho: R_{0, n} \rightarrow R_{0, n}$ be a ring homomorphism defined by

$$
\begin{aligned}
\rho\left(v_{k}\right)=f_{k} & \text { for } 0 \leq k \leq n-1, \\
\rho\left(v_{k}\right)=v_{k} & \text { for } k \geq n, \\
\rho\left(c_{n, i}\right)=c_{n, i} & \text { for } 0 \leq i \leq n-1 .
\end{aligned}
$$

Then, it is clear that for $k=0, \ldots, n-1, \rho^{p}\left(v_{k}\right)=v_{k}+p f_{k}^{\prime}=v_{k}$ where $f_{k}^{\prime}=$ $v_{n} \beta_{k, n}+v_{n+1} \beta_{k, n+1}+\cdots$. So the ring homomorphism $\rho$ is a ring automorphism. It is also clear that $v_{0}, \ldots, v_{n-1}$ is a regular sequence. Hence, $\rho\left(v_{0}\right), \ldots, \rho\left(v_{n-1}\right)$ is also a regular sequence. Thus, $f_{0}, \ldots, f_{n-1}$ is a regular sequence.

Proof of Theorem 1.8 in the case $n=1$. In the case $n=1$, the statement of Theorem 1.8 is that $f_{0}=v_{m} c_{n, 0}^{p^{m-1}+\cdots+1}+v_{m+1} c_{n, 0}^{p^{m}+p^{m-1}+\cdots+1}+\cdots$ is not a zerodivisor. It is clear that $R(m, 1)$ is an integral domain, so that Theorem 1.8 holds in the case $n=1$.

Lemma 6.1. Let $\Delta_{m}$ be the determinant of the matrix

$$
A_{m}=\left(\begin{array}{ccc}
\alpha_{0, m} & \cdots & \alpha_{0, m+n-1} \\
\vdots & \ddots & \vdots \\
\alpha_{n-1, m} & \cdots & \alpha_{n-1, m+n-1}
\end{array}\right) .
$$

Then $\Delta_{0}=1$ and

$$
\Delta_{m+1}=\Delta_{m}^{p} c_{n, 0}
$$

for $m \geq 0$. In particular, the above matrix has an inverse as a matrix of coefficients in $R_{m, n}\left[c_{n, 0}^{-1}\right]$.

This lemma will be proved in the last parts of this section.

Lemma 6.2. The ring $\left(R_{m, n} / J_{\ell}\right)\left[c_{n, 0}^{-1}\right]$ is an integral domain for $\ell=0, \ldots, n-1$. 
Proof. Let

$$
f_{i}^{\prime}=v_{m+n} \alpha_{i, m+n}+v_{m+n+1} \alpha_{i, m+n+1}+\cdots
$$

for $i=0, \ldots, n-1$. Let

$$
\rho_{m}: R_{m, n}\left[c_{n, 0}^{-1}\right] \rightarrow R_{m, n}\left[c_{n, 0}^{-1}\right]
$$

be a ring homomorphism defined by

$$
\begin{aligned}
\rho_{m}\left(v_{m+k}\right) & =v_{m+k}+f_{k}^{\prime} & & \text { for } 0 \leq k \leq n-1, \\
\rho_{m}\left(v_{m+k}\right) & =v_{m+k} & & \text { for } k \geq m+n, \\
\rho_{m}\left(c_{n, i}\right) & =c_{n, i} & & \text { for } 0 \leq i \leq n-1 .
\end{aligned}
$$

As in the above proof of Theorem 1.8 in the case $m=0, \rho_{m}^{p}=1$, so that $\rho_{m}$ is a ring automorphism.

We define a ring homomorphism

$$
\psi_{m}: R_{m, n}\left[c_{n, 0}^{-1}\right] \rightarrow R_{m, n}\left[c_{n, 0}^{-1}\right]
$$

by

$$
\begin{aligned}
\psi_{m}\left(v_{m+k}\right) & =v_{m} \alpha_{k, m}+\cdots+v_{m+n-1} \alpha_{k, m+n-1} & & \text { for } 0 \leq k \leq n-1 \\
\psi_{m}\left(v_{m+k}\right) & =v_{m+k} & & \text { for } n \leq k \\
\psi_{m}\left(c_{n, i}\right) & =c_{n, i} & & \text { for } 0 \leq i \leq n-1
\end{aligned}
$$

By Lemma 6.1 , in $R_{m, n}\left[c_{n, 0}^{-1}\right]$, the inverse of the matrix $A_{m}$ exists. Hence, $\psi_{m}$ also has an inverse, that is, $\psi_{m}$ is also a ring automorphism. Therefore, $\psi_{m} \circ \rho_{m}$ is also a ring automorphism and $\psi_{m} \circ \rho_{m}$ maps $v_{m}, \ldots, v_{m+n-1}$ to $f_{0}, \ldots, f_{n-1}$. It is clear that $\psi_{m} \circ \rho_{m}$ induces an isomorphism

$$
R_{m, n}\left[c_{n, 0}^{-1}\right] /\left(v_{m+\ell}, \ldots, v_{m+n-1}\right) \rightarrow\left(R_{m, n} / J_{\ell}\right)\left[c_{n, 0}^{-1}\right] .
$$

It is clear that $R_{m, n}\left[c_{n, 0}^{-1}\right] /\left(v_{m+\ell}, \ldots, v_{m+n-1}\right)$ is an integral domain and so is $\left(R_{m, n} / J_{\ell}\right)\left[c_{n, 0}^{-1}\right]$.

Lemma 6.3. Let $\phi: R_{m, n} \rightarrow R_{m-1, n-1}$ be a ring homomorphism defined by

$$
\begin{aligned}
\phi\left(v_{k}\right) & =v_{k-1}, \\
\phi\left(c_{n, i}\right) & =c_{n-1, i-1}, \\
\phi\left(c_{n, 0}\right) & =0 .
\end{aligned}
$$

Then, $\phi$ maps $f_{k}$ to $f_{k-1}$ for $k=1, \ldots, n-1$ and $\phi\left(f_{0}\right)=0$.

Proof. It suffices to show that

(1) $\phi\left(\alpha_{0, n}\right)=0$,

(2) $\phi\left(\alpha_{i, k}\right)=\alpha_{i-1, k-1}$ for $i \geq 1$.

It is clear from the fact that $\alpha_{0, n}=-c_{n, 0}$ for $k \geq 1$ that (1) holds. We prove this lemma by induction on $k$. For $i \geq 1$,

$$
\begin{aligned}
\phi\left(\alpha_{i, k}\right) & =\phi\left(\alpha_{i-1, k-1}^{p}-\alpha_{n-1, k-1}^{p} c_{n, k}\right) \\
& =a_{i-2, k-2}^{p}-\alpha_{n-2, k-2}^{p} c_{n-1, k-1} \\
& =\alpha_{i-1, k-1}
\end{aligned}
$$

in $R_{m-1, n-1}$.

Proposition 6.4. If Theorem 1.8 holds for $R_{m-1, n-1}$, then $c_{n, 0}$ is not a zerodivisor in $R_{m, n} / J_{\ell}$ for $1 \leq \ell \leq n-1$. 
Proof. Recall $J_{\ell}=\left(f_{\ell}, \ldots, f_{n-1}\right)$. We prove this proposition by induction on $n-1-\ell$. In the case $n-1-\ell=0$, the proposition holds because $R_{m, n}$ is an integral domain.

Suppose that

$$
c_{n, 0} a=a_{\ell} f_{\ell}+\cdots+a_{n-1} f_{n-1}
$$

in $R_{m, n}$. Then,

$$
\phi\left(a_{\ell}\right) \phi\left(f_{\ell}\right)+\cdots+\phi\left(a_{n-1}\right) \phi\left(f_{n-1}\right)=0
$$

in $R_{m-1, n-1}$. By the induction hypothesis, $\phi\left(f_{\ell}\right), \ldots, \phi\left(f_{n-1}\right)$ is a regular sequence in $R_{m-1, n-1}$. Therefore,

$$
\phi\left(a_{\ell}\right)=b_{\ell+1} \phi\left(f_{\ell+1}\right)+\cdots+b_{n-1} \phi\left(f_{n-1}\right)
$$

for some $b_{\ell+1}, \ldots b_{n-1}$. Since the kernel of $\phi$ is a principal ideal $\left(c_{n, 0}\right)$,

$$
a_{\ell}=b_{\ell+1}^{\prime} f_{\ell+1}+\cdots+b_{n-1}^{\prime} f_{n-1}+c_{n, 0} b
$$

for some $b_{k}^{\prime}, b \in R_{m, n}$ such that $\phi\left(b_{k}^{\prime}\right)=b_{k}$ for $k=\ell+1, \ldots, n-1$. From (1), (2),

$$
c_{n, 0} a=c_{n, 0} b f_{\ell}+\left(a_{\ell+1}+b_{\ell+1}^{\prime} f_{\ell}\right) f_{\ell+1}+\cdots+\left(a_{n-1}+b_{n-1}^{\prime} f_{\ell}\right) f_{n-1}
$$

in $R_{m, n}$. Hence,

$$
c_{n, 0}\left(a-b f_{\ell}\right) \equiv 0
$$

in $R_{m, n} / J_{\ell+1}$. Since $c_{n, 0}$ is not a zerodivisor, $a-b f_{\ell}=0$ in $R_{m, n} / J_{\ell+1}$. Hence, $a=0$ in $R_{m, n} / J_{\ell}$.

Remark. Since $f_{0}$ is divisible by $c_{n, 0}, c_{n, 0}$ is a zerodivisor in $R_{m, n} / J_{0}$.

Proposition 6.5. If Theorem 1.8 holds for $R_{m-1, n-1}$, then $R_{m, n} / J_{\ell}$ is an integral domain for $1 \leq \ell \leq n-1$.

Proof. For $1 \leq \ell \leq n-1$, since $c_{n, 0}$ is not a zerodivisor in $R_{m, n} / J_{\ell}$ the induced homomorphism

$$
R_{m, n} / J_{\ell} \rightarrow\left(R_{m, n} / J_{\ell}\right)\left[c_{n, 0}^{-1}\right]
$$

is a monomorphism. Since $\left(R_{m, n} / J_{\ell}\right)\left[c_{n, 0}^{-1}\right]$ is an integral domain by Lemma 6.2 , $R_{m, n} / J_{\ell}$ is also an integral domain.

This proposition completes the proof of Theorem 1.8.

Now we prove Lemma 6.1. Firstly, we prove that

$$
\operatorname{det} A_{m+1}=(-1)^{n-1} c_{n, 0} \operatorname{det} A_{m}^{p}
$$

for $m \geq n$. For the sake of notational ease, let

$$
\alpha=\alpha_{n-1, m} \cdots \alpha_{n-1, m+n-1} .
$$

Note that $\alpha_{n-1, m} \neq 0$ for $m \geq n$ from Proposition 3.6. We write $\beta_{i, j}$ for the quotient

and $\gamma_{i, j}$ for

$$
\alpha_{i, m+j} / \alpha_{n-1, m+j}
$$

where $j=1, \ldots, n-1$.

$$
\beta_{i, j}-\beta_{i, 0}
$$

By definition,

$$
\operatorname{det} A_{m}=\left|\begin{array}{cccc}
\alpha_{0, m} & \alpha_{0, m+1} & \cdots & \alpha_{0, m+n-1} \\
\vdots & \vdots & & \vdots \\
\alpha_{n-2, m} & \alpha_{n-2, m+1} & \cdots & \alpha_{n-2, m+n-1} \\
\alpha_{n-1, m} & \alpha_{n-1, m+1} & \cdots & \alpha_{n-1, m+n-1}
\end{array}\right| .
$$


Dividing the $j$-th column by $\alpha_{n-1, m+j-1}$, we have

$$
\operatorname{det} A_{m} / \alpha=\left|\begin{array}{cccc}
\beta_{0,0} & \beta_{0,1} & \cdots & \beta_{0, n-1} \\
\vdots & \vdots & & \vdots \\
\beta_{n-2,0} & \beta_{n-2,1} & \cdots & \beta_{n-2, n-1} \\
1 & 1 & \cdots & 1
\end{array}\right| .
$$

Subtracting the first column from the $j$-th column for $j=2, \ldots, n$, we have

$$
\operatorname{det} A_{m} / \alpha=\left|\begin{array}{cccc}
\beta_{0,0} & \gamma_{0,1} & \cdots & \gamma_{0, n-1} \\
\vdots & \vdots & & \vdots \\
\beta_{n-2,0} & \gamma_{n-2,1} & \cdots & \gamma_{n-2, n-1} \\
1 & 0 & \cdots & 0
\end{array}\right|
$$

Hence,

$$
\operatorname{det} A_{m}=(-1)^{n-1} \alpha\left|\begin{array}{cccc}
\gamma_{0,1} & \gamma_{0,2} & \cdots & \gamma_{0, n-1} \\
\vdots & \vdots & & \vdots \\
\gamma_{n-2,1} & \gamma_{n-2,2} & \cdots & \gamma_{n-2, n-1}
\end{array}\right|
$$

On the other hand, by Proposition 3.4, $\operatorname{det} A_{m+1}$ is equal to

$$
\left|\begin{array}{ccc}
-\alpha_{n-1, m}^{p} c_{n, 0} & \cdots & -\alpha_{n-1, m+n-1}^{p} c_{n, 0} \\
\alpha_{0, m}^{p}-\alpha_{n-1, m}^{p} c_{n, 1} & \cdots & \alpha_{0, m+n-1}^{p}-\alpha_{n-1, m+n-1}^{p} c_{n, 1} \\
\vdots & \ddots & \vdots \\
\alpha_{n-2, m}^{p}-\alpha_{n-1, m}^{p} c_{n, n-1} & \cdots & \alpha_{n-2, m+n-1}^{p}-\alpha_{n-1, m+n-1}^{p} c_{n, n-1}
\end{array}\right| .
$$

Dividing the $j$-th column by $\alpha_{n-1, m+j-1}^{p}$ for each $j=1, \ldots, n$, we have

$$
\operatorname{det} A_{m+1} / \alpha^{p}=\left|\begin{array}{cccc}
-c_{n, 0} & -c_{n, 0} & \cdots & -c_{n, 0} \\
\beta_{0,0}^{p}-c_{n, 1} & \beta_{0,1}^{p}-c_{n .1} & \cdots & \beta_{0, n-1}^{p}-c_{n, 1} \\
\vdots & \vdots & \ddots & \vdots \\
\beta_{n-2,0}^{p}-c_{n, n-1} & \beta_{n-2,1}^{p}-c_{n, n-1} & \cdots & \beta_{n-2, n-1}^{p}-c_{n, n-1}
\end{array}\right| .
$$

Subtracting the first column from the $j$-th column for $j=2, \ldots, n$, we have

$$
\operatorname{det} A_{m+1} / \alpha^{p}=\left|\begin{array}{cccc}
-c_{n, 0} & 0 & \cdots & 0 \\
\beta_{0,0}^{p}-c_{n, 1} & \gamma_{0,1}^{p} & \cdots & \gamma_{0, n-1}^{p} \\
\vdots & \vdots & \ddots & \vdots \\
\beta_{n-2,0}^{p}-c_{n, n-1} & \gamma_{n-2,1}^{p} & \cdots & \gamma_{n-2, n-1}^{p}
\end{array}\right| \text {. }
$$

Hence,

$$
\operatorname{det} A_{m+1}=-c_{n, 0} \alpha^{p}\left|\begin{array}{cccc}
\gamma_{0,1} & \gamma_{0,2} & \cdots & \gamma_{0, n-1} \\
\vdots & \vdots & \ddots & \vdots \\
\gamma_{n-2,1} & \gamma_{n-2,2} & \cdots & \gamma_{n-2, n-1}
\end{array}\right|^{p}
$$

Therefore, we have

$$
\operatorname{det} A_{m+1}=(-1)^{n} c_{n, 0}\left(\operatorname{det} A_{m}{ }^{p}\right)
$$

for $m \geq n$. 
Secondly, we deal with the case $0 \leq m \leq n-1$. It is clear from Proposition 3.5 that $\operatorname{det} A_{0}=1$. Suppose that $1 \leq m \leq n-1$. From Proposition 3.5, we have $\alpha_{i, k}=\delta_{i, k}$ for $k \leq n-1$.

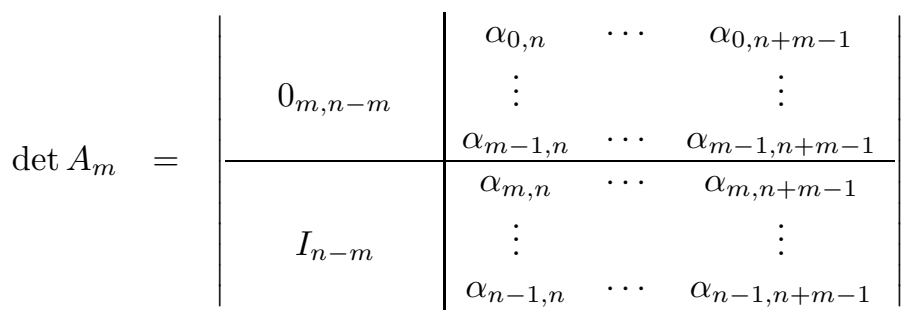

where $0_{m, n-m}$ is the $m \times(n-m)$ zero matrix and $I_{n-m}$ is the $(n-m) \times(n-m)$ identity matrix. So we get

$$
\operatorname{det} A_{m}=(-1)^{(n-1)(n-m)}\left|\begin{array}{ccc}
\alpha_{0, n} & \cdots & \alpha_{0, n+m-1} \\
\vdots & & \vdots \\
\alpha_{m-1, n} & \cdots & \alpha_{m-1, n+m-1}
\end{array}\right| .
$$

Using Proposition 3.5 and Proposition 3.6, subtracting the first column multiplied by $\alpha_{n-1, n+j-1}^{p}$ from the $j$-th column, we have

$$
\begin{aligned}
\operatorname{det} A_{m+1} & =(-1)^{(n-1)(n-m-1)}\left|\begin{array}{cccc}
-c_{n, 0} & 0 & \cdots & 0 \\
-c_{n, 1} & \alpha_{0, n}^{p} & \cdots & \alpha_{0, n+m-2}^{p} \\
\vdots & \vdots & & \vdots \\
-c_{n, m-1} & \alpha_{m-2, n}^{p} & \cdots & \alpha_{m-2, n+m-2}^{p}
\end{array}\right| \\
& =\left.(-1)^{(n-1)(n-m-1)+1} c_{n, 0}\left|\begin{array}{ccc}
\alpha_{0, n} & \cdots & \alpha_{0, n+m-2} \\
\vdots & & \vdots \\
\alpha_{m-2, n} & \cdots & \alpha_{m-2, n+m-2}
\end{array}\right|\right|^{p} \\
& =(-1)^{n} c_{n, 0}\left(\operatorname{det} A_{m}{ }^{p}\right) .
\end{aligned}
$$

This completes the proof of Lemma 6.1.

\section{REFERENCES}

[A-G-M-V] K. Andersen, J. Grodal, M. Møller and A. Viruel. The classification of p-compact groups for $p$ odd. preprint arXiv:math. AT/0302346 vl 27 Feb. (2003).

[Bo1] J.M. Boardman. Operations on the Adams spectral sequence for Brown-Peterson homology and cohomology. preprint (1988).

[Bo2] J.M. Boardman. Conditionally Convergent Spectral Sequences. Contemp. Math., 239 (1999) 49-84. MR1718076 (2000m:55024)

[Bo-Jo-Wi] J.M. Boardman, D.C. Johnson and W.S. Wilson. Unstable operations in generalized cohomology. Handbook of algebraic topology (1995) 687-828. MR1361900 (97b:55022)

[Ka-Mi] M. Kameko and M. Mimura. Mui invariant and Milnor operations. preprint (2003).

[Ka] M. Kameko. Poincaré series of cotorsion products. Preprint (2005).

[Ko-Mi] A. Kono and M. Mimura. Cohomology mod 3 of the classifying space of the Lie group $E_{6}$. Math. Scand. 46 (1978), 223-235. MR591603 (81m:55015)

[Ko-Ya] A. Kono and N. Yagita. Brown-Peterson and ordinary cohomology theories of classifying spaces for compact Lie groups. Trans. of AMS. 339 (1993), 781-798. MR1139493 (93m:55006)

[Mo-Vi] L. Molina and A. Vistoli. On the Chow rings of classifying spaces for classical groups. Rend. Sem. Mat. Univ. Padova 116 (2006), 271-298. MR2287351 
[Mu H. Mui. Modular invariant theory and the cohomology algebras of symmetric groups. J. Fac. Sci. U. of Tokyo 22 (1975), 319-369. MR0422451 (54:10440)

[Ra-Wi-Ya] D. C. Ravenel, W. S. Wilson and N. Yagita. Brown-Peterson cohomology from Morava K-theory. K-theory 15 (1998), 147-199. MR1648284 (2000d:55012)

[Te-Ya] M. Tezuka and N. Yagita. The varieties of the $\bmod p$ cohomology rings of extraspecial p-groups for an odd prime p. Math. Proc. Cambridge Phil. Soc. 94 (1983) 449-459. MR720796 (85g:20069)

[Toda] H. Toda. Cohomology mod 3 of the classifying space $B F_{4}$ of the exceptional group F. J. Math. Kyoto Univ. 13 (1973) 97-115. MR0321086 (47:9619)

[To] B. Totaro. The Chow ring of classifying spaces. Proc.of Symposia in Pure Math. "Algebraic K-theory" (1997:University of Washington, Seattle) 67 (1999), 248-281. MR1743244 (2001f:14011)

[Va-Vi] A. Vavpetič and A. Viruel. On the $\bmod p$ cohomology of BPU( $p)$. Trans. AMS 357 (2005), 4517-4532. MR2156719 (2006d:55013)

[Ve] G. Vezzosi. On the Chow ring of the classifying stack of $P G L_{3, C}$. J. Reine Angew. Math. 523 (2000), 1-54. MR1762954 (2001f:14012)

[Vi] A. Vistoli. On the cohomology and the Chow ring of the classifying space of $P G L_{p}$. arXiv:math.AG/0505052 (2005).

[Vo1] V. Voevodsky. The Milnor conjecture. www.math.unic.edu/K-theory/0170 (1996)

[Vo2] V. Voevodsky. Reduced power operations in motivic cohomology. Publ. Math. IHES 98 (2003) 1-57. MR2031198 (2005b:14038a)

[Vo3] V. Voevodsky. Motivic cohomology with $\mathbb{Z} / 2$ coefficients. Publ. Math. IHES 98 (2003) 59-104. MR2031199 (2005b:14038b)

[Ya] N. Yagita. Applications of Atiyah-Hirzebruch spectral sequences for motivic cobordism. Proc. London Math. Soc. 90 (2005) 783-816. MR2137831 (2006d:57049)

Faculty of Regional Science, Toyama University of International Studies, Toyama, JAPAN

E-mail address: kameko@tuins.ac.jp

Faculty of Education, Ibaraki University, Mito, Ibaraki, Japan

E-mail address: yagita@mx.ibaraki.ac.jp 